\title{
Isolation of a Strong Toluene Degrading Microbial Strain and Its Possible Implication for Microbial Biosensors
}

\author{
Guoce Yu, Jianlong Wang \\ Laboratory of Environmental Technology, Institute of Nuclear and New Energy Technology, Tsinghua \\ University, Beijing 100084, China \\ yugc@tsinghua.edu.cn
}

\begin{abstract}
:
Toluene is one typical monoaromatic hydrocarbon and a prevalent environmental contaminant. A bacterial strain capable of growing in and degrading high concentration of toluene was isolated. Its possible implication for constructing toluene microbial biosensors was analyzed.
\end{abstract}

Key words: toluene, biodegradation, isolation, Stenotrophomonas maltophilia, microbial biosensor

\begin{abstract}
Introduction
Toluene (together with benzene, ethylbenzene, and xylene, called BTEX) is one typical monoaromatic hydrocarbon and a prevalent environmental contaminant due to leakage and improper disposal. BTEX are toxic and often classified as carcinogens for humans. The detection of the compounds in the environment is of great importance. Toluene can be monitored by the chromatography method which is complex and costly, and yields no information on bioavailability and cell toxicity. As a complementary approach, toluene can also be detected through bioassays such as microbial biosensors which are able to show its effect on cells in a relatively rapid, inexpensive and convenient way $[1,2]$.
\end{abstract}

Construction of a microbial biosensor relies on physiological activities and genetic functions of the microorganism related. In this work, a bacterial strain capable of growing in and degrading high concentration of toluene was isolated. Aspects of its growth and toluene degradation were characterized. Possible implication by exploiting it for construction of microbial biosensors was analyzed.

\section{Materials and Methods}

A bacterial strain which can grow in mineral salts medium with toluene as the sole source of carbon and energy was isolated by selective enrichment with $0.1 \% \quad(\mathrm{v} / \mathrm{v})$ toluene from activated sludge from a waste water treatment plant in Beijing. The strain was bacilliform and Gram-negative. An analysis of the $16 \mathrm{~S}$ rRNA gene sequence and morphological and physiological characteristics showed that this is a strain of Stenotrophomonas maltophilia, and it was designated as strain YW601.

Cells were cultured at a series of initial liquid concentrations of toluene up to $401.3 \mathrm{mg} / \mathrm{L}$ in sealed $250 \mathrm{~mL}$ serum bottles, and at different temperature, $\mathrm{pH}$, oxygen supply conditions, respectively. Gas samples of toluene in the headspace were analyzed by gas chromatography, and the corresponding liquid concentrations were calculated by means of the Henry constant. Control experiments in which no microorganisms were inoculated were done simultaneously.

\section{Results and Discussion}

It is shown that the isolated strain, Stenotrophomonas maltophilia YW601, was able to grow well at initial liquid concentrations of toluene ranging from 109.5 to $401.3 \mathrm{mg} / \mathrm{L}$ with toluene as the sole source of carbon and energy in mineral salts medium (Fig. 1). The specific growth rate increased with the elevation of the toluene concentration. Toluene biodegradation by $S$. maltophilia YW601 was quite significant during the $2 \mathrm{~d}$ culture period, as seen from Table 1.

In addition, YW601 exhibited better growth at 30-37 ${ }^{\circ} \mathrm{C}$ and $\mathrm{pH}$ 6.67-8.82. Toluene concentration higher than $5 \%(\mathrm{v} / \mathrm{v})$ inhibited cell growth seriously. Moreover, YW601 was able to grow well with benzene $(0.1 \%(\mathrm{v} / \mathrm{v}))$ and ethylbenzene $(0.1 \%(\mathrm{v} / \mathrm{v}))$ as the sole carbon 
source, respectively, whereas its growth on xylenes was rather weak. (data not shown)

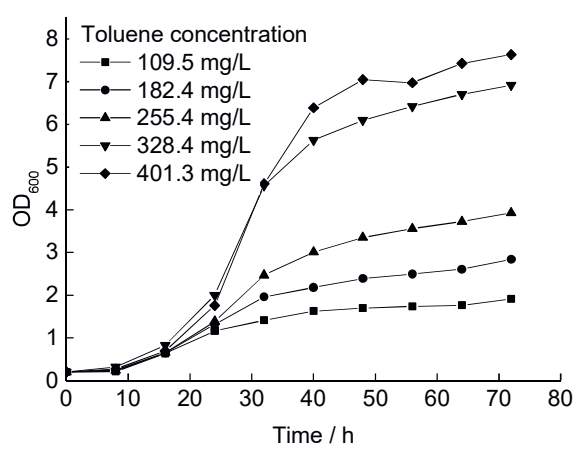

Fig. 1. Growth of Stenotrophomonas maltophilia YW601 at different initial liquid concentrations of toluene in mineral salts medium with toluene as the sole source of carbon and energy.

Tab. 1: Toluene degradation in cultures of Stenotrophomonas maltophilia YW601 with toluene as the sole source of carbon and energy in mineral salts medium (data in parenthesis represents degradation rates)

\begin{tabular}{|c|c|c|}
\hline $\begin{array}{c}\text { Time / } \\
\mathrm{h}\end{array}$ & $\begin{array}{c}\text { Liquid } \\
\text { concentration / } \\
\mathrm{mg} / \mathrm{L}\end{array}$ & $\begin{array}{c}\text { Liquid } \\
\text { concentration / } \\
\mathrm{mg} / \mathrm{L}\end{array}$ \\
\hline 0 & 182.4 & 401.3 \\
\hline 24 & $0.45(99.75 \%)$ & $58.91(85.32 \%)$ \\
\hline 48 & $0.51(99.72 \%)$ & $1.31(99.67 \%)$ \\
\hline
\end{tabular}

Microorganisms that are able to degrade toluene aerobically mainly include Pseudomonas (e.g. P. putida), Alcaligenes, Acinetobacter, Rhodococcus, and Nocardia. However, the maximum liquid concentrations of toluene which the extensively studied Pseudomonas can biodegrade are usually below 200-300 mg/L. Toluene degradation by $S$. maltophilia has rarely been reported so far and the initial liquid concentration of toluene reported was only about $30 \mathrm{mg} / \mathrm{L}$ [3]. Therefore, the S. maltophilia strain in this work exhibited outstanding capability in toluene uptake, metabolism, and tolerance. It has implications not only in toluene (and BTEX) degradation but also in toluene biosensing.

The significance of S. maltophilia YW601 in constructing microbial biosensors may be implied in the following ways: (1) A microbial biosensor may be developed for the detection of toluene based on the power of aerobic catabolism by S. maltophilia YW601. Oxygen consumption simultaneous with toluene degradation can be measured using a dissolved oxygen electrode as the transducer. The proposed biosensor is expected to have better performance compared with the case where a Pseudomonas strain was used. (2) Similarly, dependent on the catabolic ability of the strain, a toluene biosensor may be established employing redox sensitive dyes such as resazurin as an indicator of cell respiration to give a toluene-related response. (3) Genetically engineered microbial biosensors often use regulatory protein and promoter sequences from Pseudomonas cells fused with reporter genes to detect toluene. Deep understanding of metabolism and genetics of the $S$. maltophilia strain may offer better toluene biosensing elements, and also help search for new biosensor mechanisms for detecting toluene and other compounds.

\section{Conclusion}

A Stenotrophomonas maltophilia strain capable of degrading high concentration of toluene was isolated in this work. Its excellent degradation capability implies high potential for construction of microbial biosensors.

\section{References}

[1] F. Lagarde, N. Jaffrezic-Renault, Cell-based Electrochemical Biosensors for Water Quality Assessment, Analytical and Bioanalytical Chemistry 400, 947-964 (2011)

[2] R. Tecon, J. R. van der Meer, Bacterial Biosensors for Measuring Availability of Environmental Pollutants, Sensors 8, 4062-4080 (2008)

[3] E. Y. Lee, Y. S. Jun, K. -S. Cho, H. W. Ryu, Degradation Characteristics of Toluene, Benzene, Ethylbenzene and Xylene by Stenotrophomonas maltophilia T3-c, Journal of the Air \& Waste Management Association 52, 400-406 (2002) 